\title{
Roughness of the Controllability for Time Varying Systems Under the Influence of Impulses, Delay, and Nonlocal Conditions
}

https://doi.org/10.1515/msds-2020-0106

Received April 29, 2020; accepted October 3, 2020

\begin{abstract}
In this paper, we prove that the controllability of time varying linear system is preserved if we add impulses, delay and nonlocal conditions on it. In order to do that, we assume some conditions on the nonlinear terms and apply the Rothe's fixed point theorem to obtain the main result. In other words, we find sufficient controllability conditions for the semilinear system under the influence of impulses, delay, and nonlocal conditions.
\end{abstract}

Keywords: roughness, controllability, impulsive semilinear evolution equation, delay, nonlocal conditions, Rothe's fixed point theorem

MSC: Primary 34K35. Secondary 37L05

\section{Introduction}

This paper is concerned with the controllability of time varying semilinear systems under the influence of impulses, delay and nonlocal conditions as perturbations. Specifically, we shall prove that the controllability of the associated time varying linear systems is preserved by the following semilinear time varying system of differential equations with impulses, delay, and nonlocal conditions:

$$
\left\{\begin{array}{lr}
z^{\prime}(t)=A(t) z(t)+B(t) u(t)+f\left(t, z_{t}, u(t)\right), & t \in(0, \tau], \quad t \neq t_{k} \\
z(s)+g\left(z_{\tau_{1}}, z_{\tau_{2}}, \ldots, z_{\tau_{q}}\right)(s)=\phi(s), & s \in[-r, 0], \\
z\left(t_{k}^{+}\right)=z\left(t_{k}^{-}\right)+I_{k}\left(z\left(t_{k}\right), u\left(t_{k}\right)\right), & k=1,2,3, \ldots, p .
\end{array}\right.
$$

where $0<t_{1}<t_{2}<\cdots<t_{p}<\tau, 0<\tau_{1}<\tau_{2}<\cdots<\tau_{q}<\tau, z(t) \in \mathbb{R}^{n}, u(t) \in \mathbb{R}^{m}, z_{t}$ defined as a function from $[-r, 0]$ to $\mathbb{R}^{n}$ by $z_{t}(s)=z(t+s),-r \leq s \leq 0, A(t), B(t)$ are continuous matrices of dimension $n \times n$ and $n \times m$ respectively, the control function $u$ belongs to $C\left([0, \tau] ; \mathbb{R}^{m}\right), g: \mathcal{P} \mathcal{W}_{r q}\left([-r, 0] ;\left(\mathbb{R}^{n}\right)^{q}\right) \rightarrow \mathcal{P} \mathcal{W}_{r}\left([-r, 0] ; \mathbb{R}^{n}\right), \phi \in$ $\mathcal{P} \mathcal{W}_{r}\left([-r, 0] ; \mathbb{R}^{n}\right), f:[0, \tau] \times \mathcal{P} \mathcal{W}_{r}\left([-r, 0] ; \mathbb{R}^{n}\right) \times \mathbb{R}^{m} \rightarrow \mathbb{R}^{n}, I_{k} \in C\left(\mathbb{R}^{n} \times \mathbb{R}^{m} ; \mathbb{R}^{n}\right), k=1,2,3, \ldots, p$, such that

$$
\begin{gathered}
\|f(t, \phi, u)\|_{\mathbb{R}^{n}} \leq a_{0}\|\phi(-r)\|_{\mathbb{R}^{n}}^{\alpha_{0}}+b_{0}\|u\|_{\mathbb{R}^{m}}^{\beta_{0}}+c_{0}, \quad u \in \mathbb{R}^{m}, \phi \in \mathcal{P} \mathcal{W}_{r}, \quad t \in[0, \tau], \\
\left\|I_{k}\left(t_{k}, z, u\right)\right\|_{\mathbb{R}^{n} \leq} a_{k}\|z\|_{\mathbb{R}^{n}}^{\alpha_{k}}+b_{k}\|u\|_{\mathbb{R}^{m}}^{\beta_{k}}+c_{k}, \quad k=1,2,3, \ldots, p, u \in \mathbb{R}^{m}, \quad z \in \mathbb{R}^{n}, \\
\left.\|g(z)\| \leq e\|z\|_{\mathcal{P} \mathcal{W}_{r q}}^{\eta}, \quad z \in \mathcal{P} \mathcal{W}_{r q}\left([-r, 0] ;\left(\mathbb{R}^{n}\right)^{q}\right)\right),
\end{gathered}
$$

\footnotetext{
*Corresponding Author: Hugo Leiva: Yachay Tech School of Mathematical and Computational Sciences, Department of Mathematics, San Miguel de Urcuqui-100119, Imbabura - Ecuador, E-mail: hleiva@lsu.edu

Dalia Cabada, Rodolfo Gallo: Yachay Tech School of Mathematical and Computational Sciences, Department of Mathematics, San Miguel de Urcuqui-100119, Imbabura - Ecuador, E-mail: cabadadalia60@gmail.com
} 


$$
\left.\|g(z)-g(w)\| \leq K\|z-w\|_{\mathcal{P} \mathcal{W}_{r q}}, \quad z, w \in \mathcal{P} \mathcal{W}_{r q}\left([-r, 0] ;\left(\mathbb{R}^{n}\right)^{q}\right)\right),
$$

with $0<\eta \leq 1,0<\alpha_{k} \leq 1,0<\beta_{k} \leq 1, k=0,1,2,3, \ldots, p$, and

$$
z\left(t_{k}\right)=z\left(t_{k}^{+}\right)=\lim _{t \rightarrow t_{k}^{+}} z(t), \quad z\left(t_{k}^{-}\right)=\lim _{t \rightarrow t_{k}^{-}} z(t) .
$$

In order to set this problem, we shall consider the following natural Banach spaces:

The space $\mathcal{P} \mathcal{W}_{r}$ of all functions $\varphi:[-r, 0] \longrightarrow \mathbb{R}^{n}$ such that $\varphi(\cdot)$ is continuous on $[-r, 0]$ except in a finite number of point $S_{i_{\varphi}}, i=1,2, \ldots, N \leq p$ where the side limits exist $\varphi\left(S_{i_{\varphi}}^{-}\right)=\varphi\left(S_{i_{\varphi}}\right), \varphi\left(S_{i_{\varphi}}^{+}\right)$for all $i=1,2, \ldots, N$, endowed with the uniform norm denoted by $\|\cdot\|_{\mathcal{P} \mathcal{W}_{r}}$.

$$
\begin{aligned}
\mathcal{P} \mathcal{W}_{t_{1} . . t_{p}}\left([-r, \tau] ; \mathbb{R}^{n}\right)= & \left\{z:[-r, \tau] \rightarrow \mathbb{R}^{n}:\left.z\right|_{[-r, 0]} \in \mathcal{P} \mathcal{W}_{r},\left.\quad z\right|_{J} \in C\left(J^{\prime} ; \mathbb{R}^{n}\right), \exists z\left(t_{k}^{+}\right),\right. \\
& \left.z\left(t_{k}^{-}\right) \text {and } z\left(t_{k}\right)=z\left(t_{k}^{+}\right)\right\},
\end{aligned}
$$

where $J=[0, \tau]$ and $\left.J^{\prime}=J \backslash t_{1}, t_{2}, \ldots, t_{p}\right\}$, endowed with the norm

$$
\|z\|_{0}=\sup _{t \in[-r, \tau]}\|z(t)\|_{\mathbb{R}^{n}}
$$

The following notation will be used:

$$
\left(\mathbb{R}^{n}\right)^{q}=\mathbb{R}^{n} \times \mathbb{R}^{n} \times \cdots \mathbb{R}^{n}=\prod_{k=1}^{q} \mathbb{R}^{n}
$$

equipped with the norm

$$
\|z\|_{q}^{n}=\sum_{i=1}^{q}\left\|z_{i}\right\|_{\mathbb{R}^{n}}, \quad z=\left(z_{1}, z_{2}, \ldots, z_{q}\right)^{T} \in\left(\mathbb{R}^{n}\right)^{q} .
$$

Analogously, we define the space $\mathcal{P} \mathcal{W}_{r q}\left([-r, 0] ;\left(\mathbb{R}^{n}\right)^{q}\right)$ endowed with the norm

$$
\|z\|_{\mathcal{P} \mathcal{W}_{r q}}=\sup _{t \in[-r, 0]}\|z(t)\|_{q}^{n}=\sup _{t \in[-r, 0]}\left(\sum_{i=1}^{q}\left\|z_{i}(t)\right\|_{\mathbb{R}^{n}}\right), \quad \forall z \in \mathcal{P} \mathcal{W}_{r q}\left([-r, 0] ;\left(\mathbb{R}^{n}\right)^{q}\right) .
$$

On the other hand, the following Banach space is considered as well

$$
\mathcal{P} \mathcal{W}_{t_{1} . . t_{p}}\left([-r, \tau] ; \mathbb{R}^{n}\right) \times C\left([0, \tau] ; \mathbb{R}^{m}\right),
$$

equipped with the norm

$$
\left\|\left|(z, u)\|\mid=\| z\left\|_{0}+\right\| u \|_{0} .\right.\right.
$$

In $\mathbb{R}^{n} \times \mathbb{R}^{m}$ the following norm is considered:

$$
\|(z, u)\|_{1}=\|z\|_{\mathbb{R}^{n}}+\|u\|_{\mathbb{R}^{m}}=\|z\|+\|u\|, \quad \forall(z, u) \in \mathbb{R}^{n} \times \mathbb{R}^{m} .
$$

Given an arbitrary $(z, u) \in \mathcal{P} \mathcal{W}_{t_{1} . . t_{p}}\left([-r, \tau] ; \mathbb{R}^{n}\right) \times C\left([0, \tau] ; \mathbb{R}^{m}\right)$, the following quantity is also considered:

$$
\left\|f\left(\cdot, z_{t}, u\right)\right\|_{0}=\sup _{t \in[0, \tau]}\left\|f\left(t, z_{t}, u(t)\right)\right\|_{\mathbb{R}^{n}} .
$$

Also we consider the norm of $B$ as follows

$$
\|B\|=\sup _{t \in[0, \tau]}\|B(t)\|_{\mathbb{R}^{n m}}
$$

In addition, in concordance with the semilinear system (1.1), the following linear system is considered:

$$
\left\{\begin{array}{l}
z^{\prime}(t)=A(t) z(t)+B(t) u(t), \quad t \in(0, \tau], \\
z(0)=z_{0}
\end{array}\right.
$$

Now, we give the most important definition of this work, which itself is the main objective of it, to study the controllability of the semilinear system (1.1): 
Definition 1.1. (Controllability) The system (1.1) is said to be controllable on $[0, \tau]$ if for every $\phi \in$ $\mathcal{P} \mathcal{W}_{r}\left([-r, 0] ; \mathbb{R}^{n}\right), z_{1} \in \mathbb{R}^{n}$, there exists $u \in C\left([0, \tau] ; \mathbb{R}^{m}\right)$ such that the solution $z(t)$ of (1.1) verifies:

$$
z(0)+g\left(z_{\tau_{1}}, z_{\tau_{2}}, \ldots, z_{\tau_{q}}\right)(0)=\phi(0) \text { and } z(\tau)=z_{1} .
$$

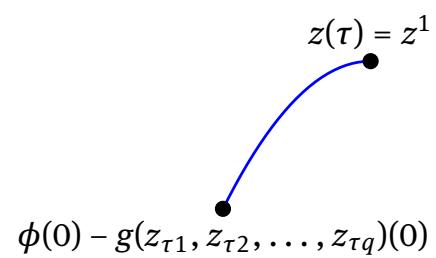

There are a lot of works about the controllability of the linear (1.6), perhaps one can see [4], [18] and [34]. Nevertheless, the reference for semilinear systems is not extensive, in this regard, we can refer to the papers done by Lukes in [27], J.C. Coron in [10](see Theorem 3.40 and Corollary 3.41 ). On the other hand, Vidyasager in [35] proved the controllability by using Schauder Fixed Point Theorem and $f$ does not depend $u \in \mathbb{R}^{m}$. However, Dauer in [13] found conditions on $f$ to prove the controllability of the semilinear system (without impulses and nonlocal conditions). But, V. N. Do [14] weaker the conditions on $f$ and prove the controllability of the system (1.1) ( without impulses, delay and non local conditions) containing Dauer's conditions; it is good to recall that all these conditions depends strongly on the linear system (1.6); especially, on the fundamental matrix $\Phi(t)$ of the linear system (2.9), which is in general not available in closed form.

There are others concepts of controllability, perhaps, the local controllability, which has been studied by [4], [5], [6], [7], [8], [9],[28],[29], and [31], but as far as we know, without impulses and non local conditions.

The controllability of differential equation with impulses is in effervescence now; for evolution equations there are papers by [30] by J.J. Nieto and C.C. Tiesdell, and in [15] by Zhi-Qing Zhu and Qing-Wen Lin. Moreover, in [22] and [23], the Rothe's fixed point theorem, which has been applied to prove the controllability of semilinear systems with impulses, is the essential motivation for doing this work.

S. Selvi and M. Malika Arjunan in [32] studied the controllability of impulsive differential systems with finite delay by using measures of noncompactness and Monch's Fixed Point Theorem. For infinite dimensional Banach spaces, we are sure that some ideas presented here can be used to address also the controllability of evolution equations with impulses, delay and nonlocal conditions simultaneously, and the nonlinear term involving all the variables, the time, the state and the control. On the other hand, some results from [3],[21], [24] and [25], can be used.

To conclude this section, we shall state Rothe's Fixed Theorem:

Theorem 1.1. (Rothe's Fixed Theorem, [1],[16], [33]) Let E be a Banach space. Let $B \subset E$ be a closed convex subset such that the zero of $E$ is contained in the interior of $B$. Let $\Psi: B \rightarrow E$ be a continuous mapping with $\Psi(B)$ relatively compact in $E$ and $\Psi(\partial B) \subset B$. Then there is a point $x^{\star} \in B$ such that $\Psi\left(x^{\star}\right)=x^{\star}$.

Our main hypotheses will be: The controllability of the linear system (1.6), the continuity of the fundamental matrix of the uncontrolled linear system and the conditions (1.2)-(1.5) satisfied by the nonlinear terms $f, g$, $I_{k}$.

\section{Controllability of Linear Systems}

In this section, we shall present some known characterization for the controllability of linear systems (1.6) without impulses, delay and nonlocal conditions. To this end, we note that for all $z_{0} \in \mathbb{R}^{n}$ and $u \in$ 
$L_{2}\left([0, \tau] ; \mathbb{R}^{m}\right)$ the initial value problem

$$
\left\{\begin{array}{l}
z^{\prime}(t)=A(t) z(t)+B(t) u(t), \quad z \in \mathbb{R}^{n}, \quad t \in[0, \tau], \\
z(0)=z_{0},
\end{array}\right.
$$

admits only one solution given by

$$
z(t)=U(t, 0) z_{0}+\int_{0}^{t} U(t, s) B(s) u(s) d s, \quad t \in[0, \tau],
$$

where $\mathcal{U}(t, s)=\Phi(t) \Phi^{-1}(s)$ and $\Phi(t)$ is the fundamental matrix of the uncontrolled linear system

$$
z^{\prime}(t)=A(t) z(t)
$$

i.e., the matrix $\Phi(t)$ satisfies:

$$
\left\{\begin{array}{l}
\Phi^{\prime}(t)=A(t) \Phi(t) \\
\Phi(0)=I_{\mathbb{R}^{n}}
\end{array}\right.
$$

where $I_{\mathbb{R}^{n}}$ is the $n \times n$ identity matrix. Therefore, there exist constants $M \geq 1$ and $\omega \geq 0$ such that

$$
\|U(t, s)\| \leq M e^{\omega(t-s)}, \quad 0 \leq s \leq t \leq \tau .
$$

Definition 2.1. For the system (1.6) we define the following concept: The controllability maps (for $\tau>0$ ) $\mathcal{G}$ : $L_{2}\left([0, \tau] ; \mathbb{R}^{m}\right) \longrightarrow \mathbb{R}^{n}$ is given by

$$
\mathcal{G} u=\int_{0}^{\tau} u(\tau, s) B(s) u(s) d s
$$

The adjoint operators $\mathcal{G}^{\star}: \mathbb{R}^{n} \longrightarrow L_{2}\left([0, \tau] ; \mathbb{R}^{m}\right)$ of the operator $\mathcal{G}$ is given by

$$
\left(\mathcal{G}^{\star} z\right)(s)=B^{\star}(s) \mathcal{U}^{\star}(\tau, s) z, \quad \forall s \in[0, \tau], \quad \forall z \in \mathbb{R}^{n} .
$$

and the Controllability Gramian operator $\mathcal{W}: \mathbb{R}^{n} \rightarrow \mathbb{R}^{n}$ is given by

$$
\mathcal{W} z=\mathcal{G G}^{\star} z=\int_{0}^{\tau} U(\tau, s) B(s) B^{\star}(s) \mathcal{U}^{\star}(\tau, s) z d s .
$$

Proposition 2.1. The systems (1.6) is controllable on $[0, \tau]$ if, and only if, $\operatorname{Ran}(\mathcal{G})=\mathbb{R}^{n}$.

Also, we shall use the following result from [11],pp 55, and [12].

Lemma 2.1. Let $Y$ and $Z$ be Hilbert space, $\mathcal{G} \in L(Y, Z)$ and $\mathcal{G}^{\star} \in L(Z, Y)$ the adjoint operator. Then the following statements holds,

(i) $\operatorname{Ran}(\mathcal{G})=Z \Longleftrightarrow \exists \gamma>0 \quad / \quad\left\|\mathcal{G}^{\star} z\right\|_{W} \geq \gamma\|z\|_{Z}, \quad z \in Z$.

(ii) $\overline{\operatorname{Ran}(\mathcal{G})}=Z \Longleftrightarrow \operatorname{ker}\left(\mathcal{G}^{*}\right)=\{0\} \Longleftrightarrow \mathcal{G}^{*}$ is $1-1$.

Lemma 2.2. (see [17]) Then the following statements are equivalent

a) $\operatorname{Ran}(\mathcal{G})=\mathbb{R}^{n}$.

b) $\operatorname{ker}\left(\mathcal{G}^{\star}\right)=\{0\}$.

c) $\exists \gamma>0 /\left\langle\mathcal{G G}^{\star} z, z\right\rangle>\gamma\|z\|^{2}, z \neq 0$ in $\mathbb{R}^{n}$.

d) $\exists \mathcal{W}^{-1} \in L\left(\mathbb{R}^{n}\right)$.

e) $B^{\star}(s) \mathcal{U}^{\star}(\tau, s) z=0, \quad \forall s \in[0, \tau] \Rightarrow z=0$. 
Therefore, the operators $Y: \mathbb{R}^{n} \rightarrow L_{2}\left([0, \tau] ; \mathbb{R}^{m}\right)$ defined by

$$
Y z=B^{\star}(\cdot) \mathcal{U}^{\star}(\tau, \cdot) \mathcal{W}^{-1} z=\mathcal{G}^{\star}(\mathcal{G G})^{\star-1} z,
$$

is called the steering operator and it is a right inverse of $\mathcal{G}$, in the sense that

$$
\mathcal{G} Y=I .
$$

Moreover,

$$
\left\|\mathcal{W}^{-1}\right\| \leq \gamma^{-1}
$$

such that

$$
\left\|\mathcal{W}^{-1} z\right\|=\left\|\left(\mathcal{G G}^{\star}\right)^{-1} z\right\| \leq \gamma^{-1}\|z\|, \quad z \in \mathbb{R}^{n},
$$

and a control steering the system (1.6) from initial state $z_{0}$ to a final state $z_{1}$ at time $\tau>0$ is given by

$$
u(t)=B^{\star}(t) \mathcal{U}^{\star}(\tau, t) \mathcal{W}^{-1}\left(z_{1}-U(\tau, 0) z_{0}\right)=Y\left(z_{1}-U(\tau, 0) z_{0}\right)(t), \quad t \in[0, \tau] .
$$

Lemma 2.3. (See [23])Let $S$ be any dense subspace of $L_{2}\left([0, \tau] ; \mathbb{R}^{m}\right)$. Then, system (1.6) is controllable with control $u \in L_{2}\left([0, \tau] ; \mathbb{R}^{m}\right)$ if, and only if, it is controllable with control $u \in S$. i.e.,

$$
\operatorname{Ran}(\mathcal{G})=\mathbb{R}^{n} \Longleftrightarrow \operatorname{Ran}\left(\left.\mathcal{G}\right|_{S}\right)=\mathbb{R}^{n},
$$

where $\left.\mathcal{G}\right|_{S}$ is the restriction of $\mathcal{G}$ to $S$.

Remark 2.1. According to the previous Lemma, if the system is controllable, it is controllable with control functions in the following dense spaces of $L_{2}\left(0, \tau ; \mathbb{R}^{m}\right)$ :

$$
S=C\left([0, \tau] ; \mathbb{R}^{m}\right), \quad S=C^{\infty}\left([0, \tau] ; \mathbb{R}^{m}\right), \quad S=\mathcal{P} \mathcal{W}\left([0, \tau] ; \mathbb{R}^{m}\right) .
$$

Moreover, the operators $\mathcal{G}, \mathcal{W}$ and $Y$ are well defined in the space of continuous functions: $\mathcal{G}: C\left([0, \tau] ; \mathbb{R}^{m}\right) \longrightarrow$ $\mathbb{R}^{n}$ by

$$
\mathcal{G} u=\int_{0}^{\tau} u(\tau, s) B(s) u(s) d s
$$

and $\mathcal{G}^{\star}: \mathbb{R}^{n} \longrightarrow C\left([0, \tau] ; \mathbb{R}^{m}\right)$ by

$$
\left(\mathcal{G}^{\star} z\right)(s)=B^{\star}(s) \mathcal{U}^{\star}(\tau, s) z, \quad \forall s \in[0, \tau] . \quad \forall z \in \mathbb{R}^{n} .
$$

Also, the Controllability Gramian operator still the same $\mathcal{W}: \mathbb{R}^{n} \rightarrow \mathbb{R}^{n}$

$$
\mathcal{W} z=\mathcal{G G}^{\star} z=\int_{0}^{\tau} \mathcal{U}(\tau, s) B(s) B^{\star}(s) \mathcal{U}^{\star}(\tau, s) z d s .
$$

Finally, the operators $Y: \mathbb{R}^{n} \rightarrow C\left([0, \tau] ; \mathbb{R}^{m}\right)$ defined by

$$
\left.Y z=B^{\star}(\cdot) \mathcal{U}^{\star}(\tau, \cdot) \mathcal{W}^{-1} z=\mathcal{G}^{\star}(\mathcal{G G})^{\star}\right)^{-1} z,
$$

is a right inverse of $\mathcal{G}$, in the sense that

$$
\mathcal{G} Y=I
$$




\section{Main Results}

In this section, we shall prove the controllability of the nonlinear system (1.1) with impulses, delay, and nonlocal conditions. To this end, for all $\phi \in \mathcal{P} \mathcal{W}_{r}\left([-r, 0] ; \mathbb{R}^{n}\right)$ and $u \in C\left([0, \tau] ; \mathbb{R}^{m}\right)$ according to [19] and [2] the initial value problem

$$
\left\{\begin{array}{lr}
z^{\prime}(t)=A(t) z(t)+B(t) u(t)+f\left(t, z_{t}, u(t)\right), & t \in(0, \tau], t \neq t_{k} \\
z(s)+g\left(z_{\tau_{1}}, z_{\tau_{2}}, \ldots, z_{\tau_{q}}\right)(s)=\phi(s), & s \in[-r, 0], \\
z\left(t_{k}^{+}\right)=z\left(t_{k}^{-}\right)+I_{k}\left(z\left(t_{k}\right), u\left(t_{k}\right)\right), & k=1,2,3, \ldots, p,
\end{array}\right.
$$

admits only one solution given by

$$
\begin{aligned}
z(t)= & U(t, 0)\left\{\phi(0)-g\left(z_{\tau_{1}}, z_{\tau_{2}}, \ldots, z_{\tau_{q}}\right)(0)\right\}+\int_{0}^{t} u(t, s) B(s) u(s) d s \\
& +\int_{0}^{t} U(t, s) f\left(s, z_{s}, u(s)\right) d s \\
& +\sum_{0<t_{k}<t} u\left(t, t_{k}\right) I_{k}\left(z\left(t_{k}\right), u\left(t_{k}\right)\right), \quad t \in[0, \tau] . \\
z(t)= & \phi(t)-g\left(z_{\tau_{1}}, z_{\tau_{2}}, \ldots, z_{\tau_{q}}\right)(t), \quad t \in[-r, 0] .
\end{aligned}
$$

Now, we define the operator

$$
\mathcal{S}: \mathcal{P} \mathcal{W}_{t_{1} . . t_{p}}\left([-r, \tau] ; \mathbb{R}^{n}\right) \times C\left([0, \tau] ; \mathbb{R}^{m}\right) \rightarrow \mathcal{P} \mathcal{W}_{t_{1} . . t_{p}}\left([-r, \tau] ; \mathbb{R}^{n}\right) \times C\left([0, \tau] ; \mathbb{R}^{m}\right)
$$

by the following formula:

$$
(y, v)=\left(\mathcal{S}_{1}(z, u), \mathcal{S}_{2}(z, u)\right)=\mathcal{S}(z, u),
$$

where $\mathcal{S}_{1}$ and $\mathcal{S}_{2}$ are defined as follows:

$$
\mathcal{S}_{1}: \mathcal{P} \mathcal{W}_{t_{1} . . t_{p}}\left([-r, \tau] ; \mathbb{R}^{n}\right) \times C\left([0, \tau] ; \mathbb{R}^{m}\right) \rightarrow \mathcal{P} \mathcal{W}_{t_{1} . . t_{p}}\left([-r, \tau] ; \mathbb{R}^{n}\right),
$$

and

$$
\mathcal{S}_{2}: \mathcal{P} \mathcal{W}_{t_{1} . . t_{p}}\left([-r, \tau] ; \mathbb{R}^{n}\right) \times C\left([0, \tau] ; \mathbb{R}^{m}\right) \rightarrow C\left([0, \tau] ; \mathbb{R}^{m}\right),
$$

such that:

$$
\begin{aligned}
& y(t)=\mathcal{S}_{1}(z, u)(t) \\
& =U(t, 0)\left\{\phi(0)-g\left(z_{\tau_{1}}, z_{\tau_{2}}, \ldots, z_{\tau_{q}}\right)(0)\right\} \\
& +\int_{0}^{t} \mathcal{U}(t, s) B(s)(Y \mathfrak{L}(z, u))(s) d s \\
& +\int_{0}^{t} U(t, s) f\left(s, z_{s}, u(s)\right) d s+\sum_{0<t_{k}<t} U\left(t, t_{k}\right) I_{k}\left(z\left(t_{k}\right), u\left(t_{k}\right)\right), \quad t \in[0, \tau], \\
& y(t)=\phi(t)-g(\ldots \ldots \ldots \ldots . .)(t), \quad t \in[-r, 0],
\end{aligned}
$$

and

$$
v(t)=\mathcal{S}_{2}(z, u)(t)=(Y \mathfrak{L}(z, u))(t)=\mathcal{B}^{*}(t) \mathcal{U}^{\star}(\tau, t) \mathcal{W}^{-1} \mathfrak{L}(z, u),
$$

with

$$
\begin{aligned}
\mathfrak{L}(z, u)=z_{1}- & U(\tau, 0)\left\{\phi(0)-g\left(z_{\tau_{1}}, z_{\tau_{2}}, \ldots, z_{\tau_{q}}\right)(0)\right\} \\
& -\int_{0}^{\tau} U(\tau, s) f\left(s, z_{s}, u(s)\right) d s \\
& -\sum_{0<t_{k}<\tau} U\left(\tau, t_{k}\right) I_{k}\left(z\left(t_{k}\right), u\left(t_{k}\right)\right) .
\end{aligned}
$$


The following proposition follows trivially from the definition of the operator $\mathcal{S}$.

Proposition 3.1. The Semilinear System (1.1) with impulses, delay, and nonlocal conditions is controllable if, and only if, for all initial state $\phi \in \mathcal{P} \mathcal{W}_{r}\left([-r, 0] ; \mathbb{R}^{n}\right)$ and a final state $z_{1}$ the operator $\mathcal{S}$ given by (3.27)-(3.29) has a fixed point. i.e.,

$$
\exists(z, u) \in \operatorname{Dom}(\mathcal{S}) \quad \text { such that } \quad \mathcal{S}(z, u)=(z, u) .
$$

Theorem 3.1. Suppose that conditions (1.2)-(1.5) hold and the linear system (1.6) is controllable on [0, $\tau]$. If $0 \leq \alpha_{k}<1,0 \leq \beta_{k}<1, \quad k=0,1,2,3, \ldots, p, 0 \leq \eta<1$, then the nonlinear system (1.1) is controllable on $[0, \tau]$. Moreover, exists a control $u \in C\left([0, \tau] ; \mathbb{R}^{m}\right)$ such that for a given $\phi \in \mathcal{P} \mathcal{W}_{r}\left([-r, 0] ; \mathbb{R}^{n}\right), z_{1} \in \mathbb{R}^{n}$ the corresponding solution $z(\cdot)$ of (1.1) satisfies:

$$
\begin{aligned}
z_{1}= & \mathcal{U}(\tau, 0)\left\{\phi(0)-g\left(z_{\tau_{1}}, z_{\tau_{2}}, \ldots, z_{\tau_{q}}\right)(0)\right\}+\int_{0}^{\tau} U(\tau, s) B(s) u(s) d s \\
& +\int_{0}^{\tau} U(\tau, s) f\left(s, z_{s}, u(s)\right) d s+\sum_{0<t_{k}<\tau} U\left(\tau, t_{k}\right) I_{k}\left(t_{k}, z\left(t_{k}\right), u\left(t_{k}\right)\right),
\end{aligned}
$$

and

$$
u(t)=B^{\star}(t) \mathcal{U}^{\star}(\tau, t) \mathcal{W}^{-1} \mathfrak{L}(z, u),
$$

with

$$
\begin{aligned}
\mathfrak{L}(z, u)= & z_{1}-U(\tau, 0)\left\{\phi(0)-g\left(z_{\tau_{1}}, z_{\tau_{2}}, \ldots, z_{\tau_{q}}\right)(0)\right\}-\int_{0}^{\tau} U(\tau, s) f\left(s, z_{s}, u(s)\right) d s \\
& -\sum_{0<t_{k}<\tau} U\left(\tau, t_{k}\right) I_{k}\left(z\left(t_{k}\right), u\left(t_{k}\right)\right) .
\end{aligned}
$$

Proof We shall prove this theorem by claims.

Claim 1. The operator $\mathcal{S}$ is continuous. In fact, to prove the continuity of $\mathcal{S}$, it is enough to prove the continuity of the operators $\mathcal{S}_{1}$ and $\mathcal{S}_{2}$ defined above.

The continuity of $\mathcal{S}_{1}$ follows from the continuity of the nonlinear functions $f\left(t, z_{s}, u\right), I_{k}(z, u), g(z)$ and the following estimate

$$
\begin{aligned}
\left\|\mathcal{S}_{1}(z, u)-\mathcal{S}_{1}(w, v)\right\| \leq & \|U(t, 0)\|\left\|\left[g\left(z_{\tau_{1}}, z_{\tau_{2}}, \ldots, z_{\tau_{q}}\right)(0)-g\left(w_{\tau_{1}}, w_{\tau_{2}}, \ldots, w_{\tau_{q}}\right)(0)\right]\right\| \\
& +\int_{0}^{t}\|U(t, s)\|\|B(s)\|\left\|B^{\star}(s)\right\|\left\|\mathcal{U}^{\star}(\tau, s)\right\|\left\|\mathcal{W}^{-1}\right\|\|\mathfrak{L}(z, u)-\mathfrak{L}(w, v)\| d s \\
& +\int_{0}^{t}\|U(t, s)\|\left\|f\left(s, z_{s}, u(s)\right)-f\left(s, w_{s}, v(s)\right)\right\| d s \\
& +\sum_{0<t_{k}<t}\left\|U\left(t, t_{k}\right)\right\|\left\|I_{k}\left(z\left(t_{k}\right), u\left(t_{k}\right)\right)-I_{k}\left(w\left(t_{k}\right), v\left(t_{k}\right)\right)\right\|, \\
\leq & M e^{\omega \tau}\|z-w\|+\frac{M^{2}}{\omega}\left[e^{2 \omega \tau}-1\right]\left\|B^{2}\right\|\left\|\mathcal{W}^{-1}\right\|\|\mathfrak{L}(z, u)-\mathfrak{L}(w, v)\| \\
& +\frac{M}{\omega}\left[e^{\omega \tau}-1\right] \sup _{s \in J}\left\|f\left(s, z_{s}, u(s)\right)-f\left(s, w_{s}, v(s)\right)\right\| \\
& +M e^{\omega \tau} \sum_{0<t_{k}<t}\left\|I_{k}\left(t_{k}, z\left(t_{k}\right), u\left(t_{k}\right)\right)-I_{k}\left(t_{k}, w\left(t_{k}\right), v\left(t_{k}\right)\right)\right\|, \\
\leq & K_{1}\|z-w\|+K_{2} \sup _{s \in J}\left\|f\left(s, z_{s}, u(s)\right)-f(s, w s, v(s))\right\|
\end{aligned}
$$




$$
+K_{3} \sum_{0<t_{k}<t}\left\|I_{k}\left(t_{k}, z\left(t_{k}\right), u\left(t_{k}\right)\right)-I_{k}\left(t_{k}, w\left(t_{k}\right), v\left(t_{k}\right)\right)\right\|,
$$

where,

$$
K_{1}=K_{4} \widehat{K}, \quad K_{2}=\frac{M}{\omega}\left(e^{\omega \tau}-1\right) \widehat{K}, \quad K_{3}=M e^{\omega \tau} \widehat{K},
$$

with,

$$
\widehat{K}=1+\frac{M^{2}}{\omega}\left(e^{2 \omega \tau}-1\right)\|B\|^{2} \gamma^{-1} \text {, and } \quad K_{4}=M e^{\omega \tau} K
$$

and,

$$
\begin{aligned}
\|\mathfrak{L}(z, u)-\mathfrak{L}(w, v)\| \leq & M e^{\omega \tau}\|z-w\| \\
& +\frac{M}{\omega}\left[e^{\omega \tau}-1\right] \sup _{s \in J}\left\|f\left(s, z_{s}, u(s)\right)-f\left(s, w_{s}, v(s)\right)\right\| \\
& +M e^{\omega \tau} \sum_{0<t_{k}<t}\left\|I_{k}\left(t_{k}, z\left(t_{k}\right), u\left(t_{k}\right)\right)-I_{k}\left(t_{k}, w\left(t_{k}\right), v\left(t_{k}\right)\right)\right\| .
\end{aligned}
$$

The continuity of the operator $\mathcal{S}_{2}$ follows from the continuity of the operators $\mathfrak{L}$ and $Y$ define above.

Claim 2. $\mathcal{S}$ maps bounded sets of $\mathcal{P} \mathcal{W}_{t_{1} . . t_{p}}\left([-r, \tau] ; \mathbb{R}^{n}\right) \times C\left([0, \tau] ; \mathbb{R}^{m}\right)$ into equicontinuous sets of $\mathcal{P} \mathcal{W}_{t_{1} . . t_{p}}\left([-r, \tau] ; \mathbb{R}^{n}\right) \times C\left([0, \tau] ; \mathbb{R}^{m}\right)$.

Consider the following equality

$$
\left\|\mathcal{S}(z, u)\left(t_{2}\right)-\mathcal{S}(z, u)\left(t_{1}\right)\right\|_{1}=\left\|\mathcal{S}_{1}(z, u)\left(t_{2}\right)-\mathcal{S}_{1}(z, u)\left(t_{1}\right)\right\|+\left\|\mathcal{S}_{2}(z, u)\left(t_{2}\right)-\mathcal{S}_{2}(z, u)\left(t_{1}\right)\right\| .
$$

Let $D \subset \mathcal{P} \mathcal{W}_{t_{1} . . t_{p}}\left([-r, \tau] ; \mathbb{R}^{n}\right) \times C\left([0, \tau] ; \mathbb{R}^{m}\right)$ be a bounded set. The equicontinuity for $\mathcal{S}(\mathcal{D})$ is given by the equicontinuity of each one of its components $\mathcal{S}_{1}(D), \mathcal{S}_{2}(D)$, which are obtained from the continuity of $U(t, s)$ and the following estimates

$$
\begin{aligned}
\left\|\mathcal{S}_{1}(z, u)\left(t_{2}\right)-\mathcal{S}_{1}(z, u)\left(t_{1}\right)\right\| \leq & \left.\left\|U\left(t_{2}, 0\right)-U\left(t_{1}, 0\right)\right\|\left\{\|\phi(0)\|+\| \mathcal{H}\left(z_{\tau_{1}}, z_{\tau_{2}}, \ldots, z_{\tau_{q}}\right)\right)(0) \|\right\} \\
& +\int_{0}^{t_{1}}\left\|\mathcal{U}\left(t_{2}, s\right)-U\left(t_{1}, s\right)\right\|\|\mathcal{B}(s)\| \|(Y \mathfrak{L}(z, u)(s) \| d s \\
& +\int_{t_{1}}^{t_{2}}\left\|\mathcal{U}\left(t_{2}, s\right)\right\|\|\mathcal{B}(s)\| \|(Y \mathfrak{L}(z, u)(s) \| d s \\
& +\int_{0}^{t_{1}}\left\|U\left(t_{2}, s\right)-U\left(t_{1}, s\right)\right\|\left\|f\left(s, z_{s}, u(s)\right)\right\| d s \\
& +\int_{t_{1}}^{t_{2}}\left\|U\left(t_{2}, s\right)\right\|\left\|f\left(s, z_{s}, u(s)\right)\right\| d s \\
& +\sum_{0<t_{k}<t_{1}}\left\|U\left(t_{2}, t_{k}\right)-U\left(t_{1}, t_{k}\right)\right\|\left\|\mathcal{J}_{k}\left(t_{k}, z\left(t_{k}\right), u\left(t_{k}\right)\right)\right\| \\
& +\sum_{t_{1}<t_{k}<t_{2}}\left\|\mathcal{U}\left(t_{2}, t_{k}\right)\right\|\left\|\mathcal{J}_{k}\left(t_{k}, z\left(t_{k}\right), u\left(t_{k}\right)\right)\right\|, \quad \forall(z, u) \in D .
\end{aligned}
$$

and

$$
\left\|\mathcal{S}_{2}(z, u)\left(t_{2}\right)-\mathcal{S}_{2}(z, u)\left(t_{1}\right)\right\| \leq\left\|\mathcal{B}^{\star}\left(t_{2}\right) \mathcal{U}^{\star}\left(\tau, t_{2}\right)-\mathcal{B}^{*}\left(t_{1}\right) \mathcal{U}^{\star}\left(\tau, t_{1}\right)\right\|\left\|\mathcal{W}^{-1} \mathfrak{L}(z, u)\right\|, \quad \forall(z, u) \in D,
$$

Since $U(t, s)$ is continuous, $\left\|U\left(t_{2}, s\right)-U\left(t_{1}, s\right)\right\|$ goes to zero as $t_{2} \rightarrow t_{1}$, and so does the sum and the integral 
from $t_{1}$ to $t_{2}$, which implies that $S_{1}(D)$ is equicontinuous. Moreover, the equicontinuity of $S_{2}(D)$ follows from the continuity of the evolution operator $U(t, s)$. Hence, $\delta$ maps bounded sets into equicontinuous sets.

Claim 3. The set $\mathcal{S}(D)$ is relatively compact. Indeed, let $D$ be a bounded subset of $\mathcal{P} \mathcal{W}_{t_{1} . . t_{p}}\left([-r, \tau] ; \mathbb{R}^{n}\right) \times$ $C\left([0, \tau] ; \mathbb{R}^{m}\right)$. By the continuity of $f, \mathfrak{L}$, and $\mathcal{J}_{k}$, it follows that

$$
\|f(\cdot, z, u)\|_{0} \leq M_{5}, \quad\left\|\mathcal{W}^{-1} \mathfrak{L}(z, u)\right\| \leq M_{6},\left\|\mathcal{J}_{k}(z, u)\right\|_{\mathbb{R}^{n}} \leq T_{k}, \quad k=1,2, \ldots, p ; \forall(z, u) \in D .
$$

where $M_{5}, M_{6}, T_{1}, T_{2}, \ldots, T_{k} \in \mathbb{R}$. Therefore, $\mathcal{S}(D)$ is uniformly bounded. Now, we consider a sequence a $\left\{\psi_{n}=\left(\psi_{1 n}, \psi_{2 n}\right): n=1,2, \ldots\right\}$ in $\mathcal{S}(D)$. Since $\left\{\psi_{2 n}: n=1,2, \ldots\right\}$ is contained in $\mathcal{S}_{2}(D) \subset C\left([0, \tau] ; \mathbb{R}^{m}\right)$ and $S_{2}(D)$ is an uniformly bounded and equicontinuous family, by Arzelà-Ascoli Theorem, we can assume, without loss of generality, that $\left\{\psi_{2 n}: n=1,2, \ldots\right\}$ converges.

On the other hand, since $\left\{\psi_{1 n}: n=1,2, \ldots\right\}$ is contained in $\mathcal{S}_{1}(D) \subset \mathcal{P W}_{t_{1} . . t_{p}}\left([-r, \tau] ; \mathbb{R}^{n}\right)$, then $\left.\psi_{1 n}\right|_{[-r, 0]}=\phi, n=1,2, \ldots$ Baring in mind that $\left\{\psi_{1 n}: n=1,2, \ldots\right\}$ is bounded and equicontinuous in $\left[0, t_{1}\right]$, we can apply Arzelà-Ascoli Theorem again to ensure the existence of a subsequence $\left\{\psi_{1 n}^{1}: n=1,2, \ldots\right\}$ of $\left\{\psi_{1 n}: n=1,2, \ldots\right\}$, which is uniformly convergent on $\left[-r, t_{1}\right]$. Now, consider the sequence $\left\{\phi_{1 n}^{1}: n=1,2, \ldots\right\}$ on the interval $\left[t_{1}, t_{2}\right]$. On this interval the sequence $\left\{\psi_{1 n}^{1}: n=1,2, \ldots\right\}$ is uniformly bounded and equicontinuous, and for the same reason, it has a subsequence $\left\{\psi_{1 n}^{2}\right\}$ uniformly convergent on $\left[-r, t_{2}\right]$. In this way, for the intervals $\left[t_{2}, t_{3}\right],\left[t_{3}, t_{4}\right], \ldots,\left[t_{p}, \tau\right]$, we see that the sequence $\left\{\phi_{1 n}^{p+1}: n=1,2, \ldots\right\}$ converges uniformly on the interval $[-r, \tau]$. Therefore, the subsequence $\left\{\psi_{n}^{p+1}=\left(\psi_{1 n}^{p+1}, \psi_{2 n}^{p+1}\right): n=1,2, \ldots\right\}$ converges in $\mathcal{S}(D)$. This means that $\overline{\mathcal{S}(D)}$ is compact, i.e., $\mathcal{S}(D)$ is relatively compact.

Claim 4. for $0 \leq \alpha_{k}<1,0 \leq \beta_{k}<1, k=0,1,2,3, \ldots, p, 0 \leq \eta<1$, the following limit holds.

$$
\lim _{\|(z, u)\| \mid \rightarrow \infty} \frac{\||\mathcal{S}(z, u) \||}{\||(z, u) \||}=0
$$

where $\left\|\left|(z, u)\|\mid=\| z\left\|_{0}+\right\| u \|_{0}\right.\right.$ is the norm in the space $\mathcal{P} \mathcal{W}_{t_{1} . . t_{p}}\left([-r, \tau] ; \mathbb{R}^{n}\right) \times C\left([0, \tau] ; \mathbb{R}^{m}\right)$. Using the conditions (1.2)-(1.5), we get that

$$
\|\mathfrak{L}(z, u)\| \leq M_{1}+M_{2}\left\{e\|z\|^{\eta}+a_{0}\|z\|^{\alpha_{0}}+b_{0}\|u\|^{\beta_{0}}+c_{0}\right\}+M_{3} \sum_{0<t_{k}<\tau}\left\{a_{k}\|z\|^{\alpha_{k}}+b_{k}\|u\|^{\beta_{k}}+c_{k}\right\},
$$

where

$$
\begin{aligned}
& M_{1}=\left\|z_{1}\right\|+M_{3}\|\phi(0)\|, \quad M_{2}=M_{3}+\frac{M}{\omega}\left(e^{\omega \tau}-1\right) \quad \text { and } \quad M_{3}=M e^{\omega \tau} . \\
& \left\|\mathcal{S}_{2}(z, u)\right\| \leq\|B\| M_{3} M_{1} \gamma^{-1}+\|B\| M_{3} M_{2} \gamma^{-1}\left\{e\|z\|^{\eta}+a_{0}\|z\|^{\alpha_{0}}+b_{0}\|u\|^{\beta_{0}}+c_{0}\right\} \\
& +\|B\| M_{3}^{2} \gamma^{-1} \sum_{0<t_{k}<\tau}\left\{a_{k}\|z\|^{\alpha_{k}}+b_{k}\|u\|^{\beta_{k}}+c_{k}\right\} .
\end{aligned}
$$

and

$$
\begin{aligned}
\left\|\mathcal{S}_{1}(z, u)\right\| \leq & M_{3}\|\phi(0)\|+\frac{M^{2}}{\omega}\left(e^{2 \omega \tau}-1\right)\|B\|^{2} \gamma^{-1} M_{1} \\
& +M_{2} \widehat{K}\left\{e\|z\|^{\eta}+a_{0}\|z\|^{\alpha_{0}}+b_{0}\|u\|^{\beta_{0}}+c_{0}\right\} \\
& +M_{3} \widehat{K} \sum_{0<t_{k}<\tau}\left\{a_{k}\|z\|^{\alpha_{k}}+b_{k}\|u\|^{\beta_{k}}+c_{k}\right\} .
\end{aligned}
$$

Therefore,

$$
\begin{aligned}
\|\mid \mathcal{S}(z, u)\| & =\left\|\mathcal{S}_{1}(z, u)\right\|+\left\|\mathcal{S}_{2}(z, u)\right\| \\
& \leq M_{4}+\left\{\|B\| M_{3} M_{2} \gamma^{-1}+M_{2} \widehat{K}\right\}\left\{e\|z\|^{\eta}+a_{0}\|z\|^{\alpha_{0}}+b_{0}\|u\|^{\beta_{0}}+c_{0}\right\}
\end{aligned}
$$




$$
+\left\{\|B\| M_{3}^{2} \gamma^{-1}+M_{3} \widehat{K}\right\} \sum_{0<t_{k}<\tau}\left\{a_{k}\|z\|^{\alpha_{k}}+b_{k}\|u\|^{\beta_{k}}+c_{k}\right\}
$$

where $M_{4}$ is given by:

$$
M_{4}=M_{3}\|\phi(0)\|+\|B\| \gamma^{-1} M_{1}\left\{M_{3}+\frac{M^{2}}{\omega}\left(e^{2 \omega \tau}-1\right)\|B\|\right\}
$$

Hence,

$$
\begin{aligned}
\frac{\||\mathcal{S}(z, u) \||}{\||(z, u) \||} & \frac{M_{4}}{\|z\|+\|u\|} \\
& +\left\{\|B\| M_{3} M_{2} \gamma^{-1}+M_{2} \widehat{K}\right\} \\
& \times\left\{e\|z\|^{\eta-1}+a_{0}\|z\|^{\alpha_{0}-1}+b_{0}\|u\|^{\beta_{0}-1}+\frac{c_{0}}{\|z\|+\|u\|}\right\} \\
& +\left\{\|B\| M_{3}^{2} \gamma^{-1}+M_{3} \widehat{K}\right\} \times \\
& \sum_{0<t_{k}<\tau}\left\{a_{k}\|z\|^{\alpha_{k}-1}+b_{k}\|u\|^{\beta_{k}-1}+\frac{c_{k}}{\|z\|+\|u\|}\right\},
\end{aligned}
$$

Consequently,

$$
\lim _{\|(z, u)\| \mid \rightarrow \infty} \frac{\||\mathcal{S}(z, u) \||}{\||(z, u) \||}=0
$$

Claim 5. The operator $\mathcal{S}$ has a fixed point. In fact, by Claim 4, we know that for a fixed $0<\rho<1$ there exists $R>0$ big enough such that

$$
\||\mathcal{S}(z, u)\||\leq \rho\||(z, u)\||, \quad\|(z, u)\|| \geq R .
$$

Hence, if we denote by $B(0, R)$ the closed ball of center zero and radius $R>0$, we get that $\mathcal{S}(\partial B(0, R)) \subset$ $B(0, R)$. Since $\mathcal{S}$ is a compact operator, $\mathcal{S}(B)$ is relatively compact in $\mathcal{P} \mathcal{W}_{t_{1} . . t_{p}}\left([-r, \tau] ; \mathbb{R}^{n}\right) \times C\left([0, \tau] ; \mathbb{R}^{m}\right)$, and maps the sphere $\partial B(0, R)$ into the interior of the ball $B(0, R)$, we can apply Rothe's fixed point theorem 1.1 to ensure the existence of a fixed point $(z, u) \in B(0, R) \subset \mathcal{P} \mathcal{W}_{t_{1} . . t_{p}}\left([-r, \tau] ; \mathbb{R}^{n}\right) \times C\left([0, \tau] ; \mathbb{R}^{m}\right)$ such that

$$
\mathcal{S}(z, u)=(z, u) .
$$

Hence, applying the Proposition 3.1, we get that the nonlinear system (1.1) is controllable on $[0, \tau]$. Moreover,

$$
u=Y \mathfrak{L}(z, u)=B^{\star}(\cdot) \mathcal{U}^{\star}(\tau, \cdot) \mathcal{W}^{-1} \mathfrak{L}(z, u),
$$

such that for a given $\phi \in C\left([-r, 0] ; \mathbb{R}^{n}\right), z_{1} \in \mathbb{R}^{n}$ the corresponding solution $z(t)=z(t, u)$ of (1.1) satisfies:

$$
\begin{aligned}
z_{1}= & \left.U(\tau, 0)\left\{\phi(0)-\mathcal{H}\left(z_{\tau_{1}}, z_{\tau_{2}}, \ldots, z_{\tau_{q}}\right)\right)(0)\right\}+\int_{0}^{\tau} U(\tau, s) \mathcal{B}(s) u(s) d s \\
& +\int_{0}^{\tau} U(\tau, s) f\left(s, z_{s}, u(s)\right) d s+\sum_{0<t_{k}<\tau} U\left(\tau, t_{k}\right) \mathcal{J}_{k}\left(t_{k}, z\left(t_{k}\right), u\left(t_{k}\right)\right) .
\end{aligned}
$$

It finishes the proof.

Now, we present another version of the previous theorem, which follows from the estimates considered in Claim 4.

Theorem 3.2. Suppose the linear system (1.6) is controllable on $[0, \tau]$. Then the nonlinear system (1.1) is controllable if one of the following statement holds: 
a) $\alpha_{0}=1, \max \left\{\alpha_{k}: k=1,2, \ldots, p\right\}<1, \max \left\{\beta_{k}: k=0,1,2, \ldots, p\right\}<1, \quad \eta<1$ and

$$
\left\{\|B\| M_{3} M_{2} \gamma^{-1}+M_{2} \widehat{K}\right\} a_{0}<1
$$

b) $\beta_{0}=1, \max \left\{\beta_{k}: k=1,2, \ldots, p\right\}<1, \max \left\{\alpha_{k}: k=0,1,2, \ldots, p\right\}<1 \quad \eta<1$ and

$$
\left\{\|B\| M_{3} M_{2} \gamma^{-1}+M_{2} \widehat{K}\right\} b_{0}<1
$$

c) $\beta_{0}=\alpha_{0}=1, \max \left\{\beta_{k}: k=1,2, \ldots, p\right\}<1, \max \left\{\alpha_{k}: k=1,2, \ldots, p\right\}<1 \quad \eta<1$ and

$$
\left\{\|B\| M_{3} M_{2} \gamma^{-1}+M_{2} \widehat{K}\right\}\left(a_{0}+b_{0}\right)<1
$$

d) $\beta_{0}=\alpha_{0}=\eta=1, \max \left\{\beta_{k}: k=1,2, \ldots, p\right\}<1, \max \left\{\alpha_{k}: k=1,2, \ldots, p\right\}<1$ and

$$
\left\{\|B\| M_{3} M_{2} \gamma^{-1}+M_{2} \widehat{K}\right\}\left(e+a_{0}+b_{0}\right)<1 .
$$

e) $\beta_{0}<1, \alpha_{0}<1, \max \left\{\beta_{k}: k=1,2, \ldots, p\right\}<1, \max \left\{\alpha_{k}: k=1,2, \ldots, p\right\}=1 \quad \eta<1$ and

$$
\left\{\|B\| M_{3}^{2} \gamma^{-1}+M_{3} \widehat{K}\right\} \sum_{k \in S_{\alpha}} a_{k}<1,
$$

where $S_{\alpha}=\left\{k: \alpha_{k}=1\right\}$.

f) $\beta_{0}<1, \alpha_{0}<1, \max \left\{\beta_{k}: k=1,2, \ldots, p\right\}=1, \max \left\{\alpha_{k}: k=1,2, \ldots, p\right\}<1 \quad \eta<1$ and

$$
\left\{\|B\| M_{3}^{2} \gamma^{-1}+M_{3} \widehat{K}\right\} \sum_{k \in S_{\beta}} b_{k}<1,
$$

where $S_{\beta}=\left\{k: \beta_{k}=1\right\}$.

g) $\beta_{0}<1, \alpha_{0}<1, \max \left\{\beta_{k}: k=1,2, \ldots, p\right\}=1, \max \left\{\alpha_{k}: k=1,2, \ldots, p\right\}=1 \quad \eta<1$ and

$$
\left\{\|B\| M_{3}^{2} \gamma^{-1}+M_{3} \widehat{K}\right\}\left(\sum_{k \in S_{\alpha}} a_{k}+\sum_{k \in S_{\beta}} b_{k}\right)<1
$$

where

$$
M_{2}=M_{3}+\frac{M}{\omega}\left(e^{\omega \tau}-1\right), \quad \widehat{K}=1+\frac{M^{2}}{\omega}\left(e^{2 \omega \tau}-1\right)\|B\|^{2} \gamma^{-1}, \text { and } \quad M_{3}=M e^{\omega \tau}
$$

Proof Let us consider any of the conditions $a$ ) $-g$ ). Then, from the estimates obtained in Claim 4, we get that

$$
\lim _{\||(z, u) \|| \rightarrow \infty} \frac{\||\mathcal{S}(z, u) \||}{\||(z, u) \||}<\rho<1
$$

Hence, there exists $R>0$ such that

$$
\||\mathcal{S}(z, u)\||\leq \rho\||(z, u)\||,\||(z, u) \||=R .
$$

Then, analogously to the previous theorem the proof of Theorem 3.2 immediately follows by applying Proposition 3.1. 


\section{Application}

In this section we shall consider an example as an application of theorem (3.1). To this end, we consider the following semilinear time varying control system with impulses, delay and nonlocal conditions

$$
\left\{\begin{array}{lr}
z^{\prime}(t)=A(t) z(t)+B(t) u(t)+f\left(t, z_{t}, u(t)\right), & t \in(0, \tau], t \neq t_{k} \\
z(s)+g\left(z_{\tau_{1}}, z_{\tau_{2}}, \ldots, z_{\tau_{q}}\right)(s)=\phi(s), & s \in[-r, 0], \\
z\left(t_{k}^{+}\right)=z\left(t_{k}^{-}\right)+I_{k}\left(z\left(t_{k}\right), u\left(t_{k}\right)\right), & k=1,2,3, \ldots, p,
\end{array}\right.
$$

where $A(t)=a(t) A, B(t)=b(t) B$ with $A$ and $B n \times n$ and $n \times m$ constant matrices, respectively.

$a \in L^{1}[0, \tau], b \in C[0, \tau]$ and

$$
\int_{0}^{\tau} a(s) d s \neq 0, \quad b(t) \neq 0, \quad t \in[0, \tau]
$$

From [26] , we know that the Kalman’s rank conditions

$$
\operatorname{Rank}\left[B ; A B ; \cdots ; A^{n-1} B\right]=n,
$$

if, and only if, the time-varying linear system given by

$$
z^{\prime}(t)=A(t) z(t)+B(t) u(t), \quad t \in[0, \tau]
$$

with $A(t)=a(t) A, B(t)=b(t) B$, is exactly controllable on $[0, \tau]$.

In this example we shall assume that the Kalman's rank conditions holds. The nonlinear terms and the impulsive functions are all given as follows

$f:[0, \tau] \times \mathcal{P} \mathcal{W}_{r}\left([-r, 0] ; \mathbb{R}^{n}\right) \times \mathbb{R}^{m} \rightarrow \mathbb{R}^{n}$,

$$
f(t, \phi, u)=\left(\begin{array}{ccc}
\sqrt[3]{\|u\|+1} & \cdot & \sqrt[3]{\phi_{1}(-r)} \\
\sqrt[3]{\|u\|+1} & \cdot & \sqrt[3]{\phi_{2}(-r)} \\
\vdots & \cdot & \vdots \\
\sqrt[3]{\|u\|+1} & \cdot & \sqrt[3]{\phi_{n}(-r)}
\end{array}\right),
$$

$g: \mathcal{P} \mathcal{W}_{r q}\left([-r, 0] ;\left(\mathbb{R}^{n}\right)^{q}\right) \rightarrow \mathcal{P} \mathcal{W}_{r}\left([-r, 0] ; \mathbb{R}^{n}\right)$,

given by

$$
g\left(\phi_{1}, \phi_{2}, \cdots, \phi_{1}\right)=\sum_{i=1}^{q}\left(\begin{array}{c}
\sin \left(\phi_{i 1}\right) \\
\sin \left(\phi_{i 2}\right) \\
\vdots \\
\sin \left(\phi_{i n}\right)
\end{array}\right),
$$

$I_{k}: \mathbb{R}^{n} \times \mathbb{R}^{m} \rightarrow \mathbb{R}^{n}, k=1,2, \cdots, p$,

given by

$$
I_{k}(z, u)=\cos (\sqrt{\|u\|+1})\left(\begin{array}{c}
\sin \left(z_{1}^{k}\right) \\
\sin \left(z_{2}^{k}\right) \\
\vdots \\
\sin \left(z_{n}^{k}\right)
\end{array}\right),
$$

Then

$$
\|f(t, \phi, u)\| \leq \sqrt{n}\|\phi(-r)\|^{2 / 3}+2 \sqrt{n}\|u\|^{2 / 3}+2 \sqrt{n},
$$

and since $g$ and $I_{k}, k=1,2, \cdots, p$ are bounded, the conditions (1.2)-(1.5) are satisfied. Hence, the system (4.30) is exactly controllable on $[0, \tau]$. 


\section{Final Remark}

Some of the ideas presented in this work can be used to prove that under certain conditions the controllability of many control systems is not destroyed if the system is influenced by impulses, nonlocal conditions and delay simultaneously. In other words, we proved that the controllability is robust in the presence of impulses, delay, and nonlocal conditions. This happens for many real life control systems where impulses, delay, and nonlocal conditions are intrinsic phenomena of the system. Moreover, in several papers we have shown that the influence of impulses do not destroy the controllability of some known systems like the heat equation, the wave equation and the strongly damped wave equation([3, 23-25]).

Acknowledgement: This work has been supported by Yachay Tech and ULA

\section{References}

[1] J. Banas and K. Goebel, Measures of Noncompactness in Banach Spaces. Lecture Notes in Pure and Applied Mathematics, 60. Marcel Dekker, Inc., New York, 1980.

[2] D. Cabada, R. Gallo and Hugo Leiva, Existence of Solutions of Semilinear Time Varying Differential Equations with Impulses, delay and Nonlocal Conditions. Article submitted for publication.

[3] A. Carrasco, Hugo Leiva, J.L. Sanchez and A. Tineo M, Approximate Controllability of the Semilinear Impulsive Beam Equation with Impulses. Transaction on IoT and Cloud Computing 2(3) 70-88, 2014.

[4] E. N. Chukwu, Stability and Time-Optimal Control of Hereditary Systems, Mathematics in Science and Engineering, Vol. 188, Academic Press, INC., 1992.

[5] E. N. Chukwu, Nonlinear Delay Systems Controllability, Math. Anal. Appl. 162 (1991) 564- 576.

[6] E. N. Chukwu, Global Null Controllability of Nonlinear Delay Equations with Controls in a Compact Set, Optim. Theo. Appl. 53 (1987) 43-57.

[7] E. N. Chukwu, Controllability of Delay Systems with Restrained Controls, Optim. Theo. Appl. 29 (1979) 301-320.

[8] E. N. Chukwu, On the Null-Controllability of Nonlinear Delay Systems with Restrained Controls, Math. Anal. Appl. 76 (1980) 283-296.

[9] E. N. Chukwu, Null Controllability in Function Space of Nonlinear Retarded Systems with

[10] J.-M. Coron, Control and Nonlinearity. Vol. 136 of Mathematical Surveys and Monographs. American Mathematical Society, Provience, RI, 2007.

[11] R.F. curtain and A.J. Pritchard, Infinite Dimensional Linear Systems. Lecture Notes in Control and Information Sciences, 8. Springer Verlag, Berlin (1978).

[12] R.F. Curtain and H.J. Zwart, An Introduction to Infinite Dimensional Linear Systems Theory. Text in Applied Mathematics, 21. Springer Verlag, New York (1995).

[13] J.P. Dauer“Nonlinear Perturbation of Quasilinear Control Systems”. J. Math. Anal. Appl. 54 (1976), 3, 717-725.

[14] V.N. Do “Controllability of Semilinear Systems". J. Optim. Theory Appl.65 (1990),1,41-52.

[15] Zhi-Qing Zhu and Qing-Wen Lin "Exact Controllability of Semilinear Systems with Impulses". Bulletin of Math. Anal. and Appl., Vol. 4, Issues 1 (2012), pg. 157-167.

[16] G. Isac, "On Rothe's fixed point theorem in General Topological Vector Space", An. St. Univ. Ovidius Constanta, Vol. 12(2), 2004, 127-134.

[17] E. Iturriaga and H. Leiva,"A Characterization of Semilinear Surjective Operators and Applications to Control Problems”. Applied Mathematics, 2010,1,137-145.

[18] E. B. Lee, L. Markus, Foundations of Optimal Control Theory, Wiley, New York, 1967.

[19] Hugo Leiva, Karakostas Fixed Point Theorem and the Existence of Solutions for Impulsive Semilinear Evolution Equations with delay and Nonlocal Conditions. Communications inMathematicalAnalysis, Volume 21, Number 2, pp. 68-91 (2018), ISSN 1938-9787

[20] Hugo Leiva and Raul Rojas, Controllability of Semilinear Nonautonomous Systems with Impulses and Nonlocal Conditions. Equilibrium: Journal of Natural Sciences, Volumen 1, abril 2016 ISSN: 2470-1998.

[21] Hugo Leiva, "Rothe's fixed point theorem and Controllability of Semilinear Nonautonomous Systems". System and Control Letters 67 (2014) 14-18.

[22] Hugo Leiva, Rothe's fixed point theorem and Controllability of Semilinear Nonautonomous Systems. System and Control Letters 67 (2014) 14-18.

[23] Hugo Leiva, Controllability of Semilinear Impulsive Nonautonomous Systems, International Journal of Control, 2014, http/dx.doi.org/10.1080/00207179.2014.966759. 
[24] Hugo Leiva and N. Merentes,Approximate Controllability of the Impulsive Semilinear Heat Equation. Journal of Mathematics and Applications, N0. 38, pp 85-104 (2015)

[25] Hugo Leiva, Approximate Controllability of Semilinear Impulsive Evolution Equations, Abstract and Applied Analysis, Vol. 2015, Article ID 797439, 7 pages

[26] Hugo Leiva and H. Zambrano, "Rank condition for the controllability of a linear time-varying system." International Journal of Control 72.10 (1999): 929-931.

[27] D.L. LukesS, “Global Controllability of Nonlinear Systems”. SIAM J. Control Optim. 10 (1972), 1, 112-126, Erratum 11 (1973), $1,186$.

[28] K. B. Mirza, B. F. Womack, On the Controllability of Nonlinear Time-Delay Systems, IEEE Trans. Auto. Cont. Short Papers (1972) 812-814.

[29] A. S. C. Sinha, Null-Controllability of Non-Linear Infinite Delay Systems with Restrained Controls, Int. J. Cont. 42 (1985) 735 741.

[30] J.J. Nieto, C. Tisdell, On exact controllability of first-order impulsive differential equations. Advances in Difference Equations 2010, art. no. 136504, 9 pages

[31] A. S. C. Sinha, C. F. Yokomoto, Null Controllability of a Nonlinear System with Variable Time Delay, IEEE Trans. Auto. Cont. AC-25 (1980) 1234-1236.

[32] S. Selvi and M. Mallika Arjunan, “Controllability results for Impulsive Differential Systems with finite Delay". Journal of Nonlinear Science and Applications. 5(2012), 206-219.

[33] J. D.R. Smart, Fixed Point Theorems. Cambridge University Press (1974).

[34] E. D. Sontag, Mathematical Control Theory: Deterministic Finite Dimensional Systems, Springer, New York, NY, USA, 2nd edition, 1998

[35] M. Vidyasager, “A Controllability Condition for Nonlinear Systems”. IEEE Trans. Automat. Control. AC-17(1972), 5, 569-570. 